Open Access

\title{
On equilibrium configurations of nematic liquid crystals droplet with anisotropic elastic energy
}

\author{
Dong $A n^{1}$, Wei Wang ${ }^{2}$ and Pingwen Zhang ${ }^{1 *}$
}

${ }^{*}$ Correspondence:

pzhang@pku.edu.cn

${ }^{1}$ LMAM and School of

Mathematical Science, Peking

University, Beijing 100871, China

Full list of author information is

available at the end of the article

\begin{abstract}
We investigate the effect of anisotropic elastic energy on defect patterns of liquid crystals confined in a three-dimensional spherical domain within the framework of Landau-de Gennes model. Two typical strong anchoring boundary conditions, namely homeotropic and mirror-homeotropic anchoring conditions, are considered. For the homeotropic anchoring, we find three different configurations: uniaxial hedgehog, ring and split-core, in both cases with or without the anisotropic energy. For the mirror-homeotropic anchoring, there are also three analogue solutions: the uniaxial hyperbolic hedgehog, ring and split-core for the isotropic energy case. However, when the anisotropic energy is taken into account, the numerical results and rigorous analysis reveal that the uniaxial hyperbolic hedgehog is no longer a solution. Indeed, we find ring solution only for negative $L_{2}$ (the elastic coefficient of the anisotropic energy), while both split-core and ring solutions can be stable minimizers for positive $L_{2}$. More precisely, the uniaxial hyperbolic hedgehog for $L_{2}=0$ bifurcates to a split-core solution when $L_{2}$ increases and to a ring solution when $L_{2}$ decreases. This example shows that the anisotropic energy may significantly affect the symmetry of point defects with degree -1 whenever it is introduced.
\end{abstract}

\section{Background}

Configurations and defect patterns of nematic liquid crystals, subject to some topological constraints, remain to be one of the most attractive topics among research on liquid crystals, as predicting defect patterns is important both in practical and in theoretical points of view [4]. There are lots of studies on configurations and structures of defects in liquid crystals by various different mathematical models, such as Oseen-Frank model [15], Ericksen's model [5] and Landau-de Gennes (LdG) model [7,10,18]. The first two models postulate that one director preferred by molecules at each point, while the Landau-de Gennes theory allows the molecular orientation to have two preferred directions at each point. Since the Landau-de Gennes theory can capture biaxial behavior of liquid crystals near defect points, there are many studies on the defect patterns under this framework, see $[11,16-21]$ and the references therein.

(c) The Author(s) 2017. This article is distributed under the terms of the Creative Commons Attribution 4.0 International License (http://creativecommons.org/licenses/by/4.0/), which permits unrestricted use, distribution, and reproduction in any medium, provided you give appropriate credit to the original author(s) and the source, provide a link to the Creative Commons license, and indicate if changes were made. 
In the Landau-de Gennes model, the state of liquid crystals is described by a matrixvalued function $Q \in \mathcal{S}_{0}$ often referred as $Q$-tensor, where

$$
\mathcal{S}_{0}=\left\{Q \in \mathbb{R}^{3 \times 3}: Q=Q^{\mathrm{T}}, \operatorname{tr}(Q)=0\right\} .
$$

If all eigenvalues of $Q$ are equal, then $Q=0$ and it is called isotropic. If $Q$ has two equal nonzero eigenvalues, $Q$ can be written as $Q=s\left(\mathbf{n} \otimes \mathbf{n}-\frac{1}{3} I\right)$ with $s \in \mathbb{R}$ and $\mathbf{n} \in \mathbb{S}^{2}$. In this case, we call $Q$ uniaxial. If in addition $s>0$ (or $s<0$ ), we call $Q$ positive uniaxial (or negative uniaxial). When $Q$ has three distinct eigenvalues, it is called biaxial.

The Landau-de Gennes free energy functional is given by

$$
F[Q]=\int_{\Omega} f_{b}(Q)+f_{e}(Q) \mathrm{d} \mathbf{x},
$$

where $f_{b}(Q)$ is the bulk energy density

$$
f_{b}(Q)=\frac{A}{2} \operatorname{tr}\left(Q^{2}\right)-\frac{B}{3} \operatorname{tr}\left(Q^{3}\right)+\frac{C}{4} \operatorname{tr}\left(Q^{2}\right)^{2}
$$

and $f_{e}(Q)$ is the elastic energy density

$$
f_{e}(Q)=\frac{L_{1}}{2} Q_{i j, k} Q_{i j, k}+\frac{L_{2}}{2} Q_{i j, j} Q_{i k, k}+\frac{L_{3}}{2} Q_{i j, k} Q_{i k, j} .
$$

Here, we use the Einstein summation convention that we take summation over repeated indices. The commas indicate spatial derivatives. $A, B, C$ are constants depending on temperature and materials, and $L_{1}, L_{2}$ and $L_{3}$ are elastic constants. The first term in the elastic energy is called isotropic elastic energy, and the latter two are called anisotropic elastic energy terms.

From mathematical viewpoint, the presence of anisotropic energy will bring analytical difficulty due to its asymmetric structure; thus, some powerful tools, such as maximum principle, cannot be used to study the minimizers or equilibrium solutions. A well-known example is that the minimizers of isotropic Oseen-Frank energy, which are harmonic maps, have only finite singular points in three-dimensional domain, while it is quite difficult to prove a similar result for minimizers of anisotropic Oseen-Frank energy [9]. The same difficulty occurs in studying related problems within the Landau-de Gennes model. Therefore, in many existing studies, the elastic energy is assumed to be isotropic, which is referred as one-constant approximation. However, there are few concrete liquid crystal materials that have isotropic elastic coefficients. Hence, it becomes important to understand whether the anisotropic energy could affect the static or dynamic behaviors of liquid crystals. A typical example arises from the isotropic-nematic interface problem, in which it is found that whether the elastic energy is isotropic or anisotropic corresponds to different boundary conditions on the interface [6].

In this paper, we study how anisotropic energy affects the configuration with certain given boundary conditions within the framework of the Landau-de Gennes model, by combining numerical simulations and theoretical analysis. In particular, we focus on static equilibrium configurations of liquid crystals confined in a three-dimensional ball with strong anchoring conditions at the boundary.

The paper is organized as follows: In Sect. 2, we introduce the scaling and boundary conditions. In Sect. 3, we state the numerical methods we implement, followed by our 
main numerical results in Sect. 4. Section 5 presents a preliminary theoretical analysis on the behavior of uniaxial solutions under the mirror-homeotropic boundary condition. Finally, we summarize and discuss our results in Sect. 6, along with some open problems.

\section{Models and boundary conditions}

\subsection{Models and scaling}

First, we nondimensionalize the Landau-de Gennes energy (2)-(4) as in [10]. It is not hard to check that the $L_{3}$ term only differs from the $L_{2}$ term by a null Lagrangian (see [9] for a similar proof for the Oseen-Frank energy), so we assume $L_{3}=0$ for simplicity. Introduce the following parameters:

- effective temperature: $t=\frac{27 A C}{B^{2}}$,

- characteristic length: $\xi_{0}=\frac{\sqrt{27 C L_{1}}}{B}$,

- "normalized" elastic constant: $\varepsilon=\frac{\xi_{0}}{R}=\frac{\sqrt{27 C L_{1}}}{B R}$,

- anisotropic rate: $L_{21}=\frac{L_{2}}{L_{1}}$,

and let

$$
\tilde{x}=\frac{x}{R}, \quad \tilde{Q}=\sqrt{\frac{27 C^{2}}{2 B^{2}}} Q, \quad \tilde{F}=\varepsilon^{3} \sqrt{\frac{27 C^{3}}{4 B^{2} L_{1}^{3}}} F .
$$

We drop the tildes and then obtain the nondimensionalized energy functional

$$
F[Q]=\int_{\Omega}\left\{\frac{t}{2} \operatorname{tr}\left(Q^{2}\right)-\sqrt{6} \operatorname{tr}\left(Q^{3}\right)+\frac{1}{2} \operatorname{tr}\left(Q^{2}\right)^{2}+\frac{\varepsilon^{2}}{2} Q_{i j, k} Q_{i j, k}+\frac{\varepsilon^{2}}{2} L_{21} Q_{i j, j} Q_{i k, k}\right\} \mathrm{d} x .
$$

In addition, we work on the unit three-dimensional ball $\Omega=B_{1}(0)$. Minimizing (5) subject to boundary conditions given below, we will find stable configurations.

\subsection{Boundary conditions}

There are several different ways to determine boundary conditions. One of the most physical boundary conditions is the Dirichlet boundary condition, also referred as strong anchoring condition in the literature. It prescribes the value of the order tensor $Q$ on the boundary. In particular, the order tensor is often given to be a global minimizer of bulk energy, that is,

$$
\left.Q\right|_{\partial \Omega}=s_{+}\left(\mathbf{n}_{b} \otimes \mathbf{n}_{b}-\frac{I}{3}\right),
$$

with $s_{+}=\sqrt{\frac{3}{2}} \frac{(3+\sqrt{9-8 t})}{8}$ and $\mathbf{n}_{b}$ being a unit vector field on the boundary $\partial \Omega$.

When $\mathbf{n}_{b}$ is simply taken as the normal vector of the boundary, the boundary condition is called homeotropic anchoring. The nematics confined in a ball $B_{R}(0):=\{|\mathbf{x}| \leq R\}$ with homeotropic anchoring boundary condition is an important example to understand the local structures and locations of point defects with degree +1 in dimension three. There are many studies on the profiles of the solutions as well as their stabilities both theoretically and numerically $[3,8,13,14,18,20]$ in this setting. It is known that one can find a radially symmetric uniaxial solution, which is explicitly given by 


$$
Q(\mathbf{x})=s(|\mathbf{x}|)\left(\frac{\mathbf{x}}{|\mathbf{x}|} \otimes \frac{\mathbf{x}}{|\mathbf{x}|}-\frac{I}{3}\right), \quad \mathbf{x} \in B_{R}(0) .
$$

This solution is called radial hedgehog solution, which contains an isolated point defect at the center of the ball. If the temperature is sufficiently low, it has been proved that the hedgehog solution will be unstable [18], and the so-called ring solution will be more energetically favored [10]. Another solution called split-core solution has also been found and shown to be meta-stable [10].

In this paper, we are also interested in another type of strong anchoring boundary condition on $\partial B_{R}(0)$ :

$$
Q_{b}=s_{+}\left(\mathbf{n}_{b} \otimes \mathbf{n}_{b}-\frac{I}{3}\right), \mathbf{n}_{b}=\frac{1}{R}(x, y,-z),
$$

referred as mirror-homeotropic anchoring boundary condition. The motivation of assigning such anchoring condition is to model the point defects with topological degree -1 . This kind of boundary condition has also been considered in [12] to study the stability of the following elementary defect

$$
\mathbf{n}(\mathbf{x})=\frac{1}{r}(x, y,-z)
$$

of the Oseen-Frank theory with $k_{1}=k_{3}$.

The homeotropic and mirror-homeotropic anchoring can be regarded as two special cases of a family of boundary conditions. Let $(r, \theta, \varphi)$ be the usual spherical coordinate in three-dimensional space with $\theta \in[0, \pi], \varphi \in[0,2 \pi)$. We can define the following boundary conditions for all $k \in \mathbb{Z}$ :

$$
Q^{(k)}(R, \theta, \varphi)=s_{+}\left(\mathbf{n}^{(k)}(R, \theta, \varphi) \otimes \mathbf{n}^{(k)}(R, \theta, \varphi)-\frac{I}{3}\right)
$$

with

$$
\mathbf{n}^{(k)}(R, \theta, \varphi)=P_{\varphi}(\sin (k \theta), 0, \cos (k \theta))^{\mathrm{T}},
$$

and

$$
P_{\varphi}=\left(\begin{array}{ccc}
\cos \varphi & -\sin \varphi & 0 \\
\sin \varphi & \cos \varphi & 0 \\
0 & 0 & 1
\end{array}\right)
$$

The cases for $k=1$ and $k=-1$ correspond to the homeotropic and mirror-homeotropic anchoring, respectively. In "Appendix," we also present some numerical results on the equilibrium configurations subject to boundary conditions with $|k| \geq 2$. 


\section{Method for numerical simulation}

The numerical algorithm we implement in this paper is the same as that in [10], which is a spectral method based on Zernike polynomial expansion with high accuracy [22]. To explain the algorithm, we first assume

$$
Q=\left(\begin{array}{ccc}
q_{1} & q_{2} & q_{3} \\
q_{2} & q_{4} & q_{5} \\
q_{3} & q_{5} & -\left(q_{1}+q_{4}\right)
\end{array}\right)
$$

and expand $q_{i}$ into Zernike polynomials

$$
q_{i}(r, \theta, \phi)=\sum_{m=1-M}^{M-1} \sum_{l=|m|}^{L-1} \sum_{n=l}^{N-1} A_{n l m}^{(i)} Z_{n l m}(r, \theta, \phi),
$$

where $N \geq L \geq M \geq 0$, and

$$
Z_{n l m}(r, \theta, \phi)=R_{n}^{(l)}(r) Y_{l m}(\theta, \phi)
$$

with

$$
\begin{aligned}
R_{n}^{(l)}(r) & = \begin{cases}\sum_{s=0}^{(n-l) / 2} N_{n l s} r^{n-2 s}, & \frac{n-l}{2} \geq 0, \frac{n-l}{2} \in \mathbb{Z} ; \\
0, & \text { others, }\end{cases} \\
N_{n l s} & =(-1)^{s} \sqrt{2 n+3} \prod_{i=1}^{n-l}(n+l-2 s+1+i) \prod_{i=1}^{l}\left(\frac{n-l}{2}-s+i\right) \frac{2^{l-n}}{s !(n-s) !} .
\end{aligned}
$$

$Y_{l m}(\theta, \phi)=P_{l}^{|m|}(\cos \theta) X_{m}(\phi)$ are the spherical harmonic functions,

$$
X_{m}(\phi)= \begin{cases}\cos (m \phi), & m \geq 0 \\ \sin (|m| \phi), & m<0\end{cases}
$$

$P_{l}^{m}(x)(m \geq 0)$ are the normalized associated Legendre polynomials. Given coefficients $A_{n l m}^{(i)}$, the orthogonal relations of the Zernike polynomials provide us with the gradient information, which allows us to implement gradient optimization method such as BFGS [1] to determine $A_{n l m}^{(i)}$ minimizing the total energy.

To apply particular boundary conditions, we adopt the penalty function method, i.e., we introduce to the energy density function an additional term $\eta F_{p}$, in which $\eta$ is the penalty coefficient and

$$
F_{p}=\int_{\partial \Omega} f_{p} \mathrm{~d} S,
$$

where $f_{p}$ is the penalty energy density function like

$$
f_{p}(x)=\sum_{i=1}^{5}\left(q_{i}(x)-q_{i}^{+}(x)\right)^{2} .
$$


Therefore, $f_{p}$ is quadratic and reaches 0 when $Q$ is strictly subordinated to the boundary condition. We can expect that the numerical solutions will obey the boundary condition well if $\eta$ is large enough.

To obtain numerical results both efficiently and accurately enough, we start with small $N, L, M$ and gradually increase some or all of them until the numerical results converge, i.e., no significant change in the value of free energy.

To visualize biaxiality and characterize different defect patterns of liquid crystals, following [10] we define a function which characterizes the biaxiality of liquid crystals:

$$
\beta=1-6 \frac{\left(\operatorname{tr} Q^{3}\right)^{2}}{\left(\operatorname{tr} Q^{2}\right)^{3}}
$$

It is not difficult to show that $0 \leq \beta \leq 1, \beta=0$ when $Q$ is uniaxial and $\beta \neq 0$ when $Q$ is biaxial. Moreover, $\beta=1$ when $Q$ has exact two nonzero eigenvalues opposite to each other.

To detect the locations of defects, following [10] we define

$$
c_{l}=\frac{\lambda_{3}-\lambda_{2}}{\lambda_{3}-\lambda_{1}},
$$

in which $\lambda_{1} \leq \lambda_{2} \leq \lambda_{3}$ are the eigenvalues of $Q$. At defects, $c_{l}=0$, so a small positive value of $c_{l}$ is a good indicator of the locations of defects.

\section{Numerical results}

\subsection{Homeotropic anchoring condition}

When $L_{21}=0$, as having been well studied in [10], there exist three different kinds of solutions called radial hedgehog, ring disclination and split-core, which are described as follows and illustrated in Fig. 1.

- Radial hedgehog solution: A uniaxial state with $Q$ satisfying the profile (7). The center of the ball is the isolated isotropic point. Hedgehog solution is stable only for large $t$ and $\varepsilon$. For small $t$ and $\varepsilon$, the isotropic point broadens into a biaxial ring.

- Ring disclination: A biaxial state containing a ring which is a combination of point defects with degree $+1 / 2$. Around the ring is shelled by a strong biaxial region. This solution is rotationally symmetric. For small $t$ and $\varepsilon$, ring solution is more energetically favored than radial hedgehog solution.

- Split-core solution: A biaxial state containing a short +1 disclination line connecting two isotropic points. This solution is also rotationally symmetric. It seems to be metastable for all considered parameters, i.e., the ring solution or radial hedgehog solution always has lower free energy under the same parameters.

We further perform some numerical simulations with $L_{21} \neq 0$. The results suggest that there is no essential difference between cases with and without anisotropy energy. In other words, the possible stable states remain to be hedgehog and ring. The split-core solution is also obtained as a meta-stable solution.

- There still exists a radial hedgehog solution which satisfies the profile (7), but the scalar functions $h$ for $L_{21} \neq 0$ are different from those in $L_{21}=0$ (see Fig. 2). The radial hedgehog solution has been studied in [8], in which the stability/instability for different $L_{21}$ are discussed when $t$ and $\varepsilon$ are small. 


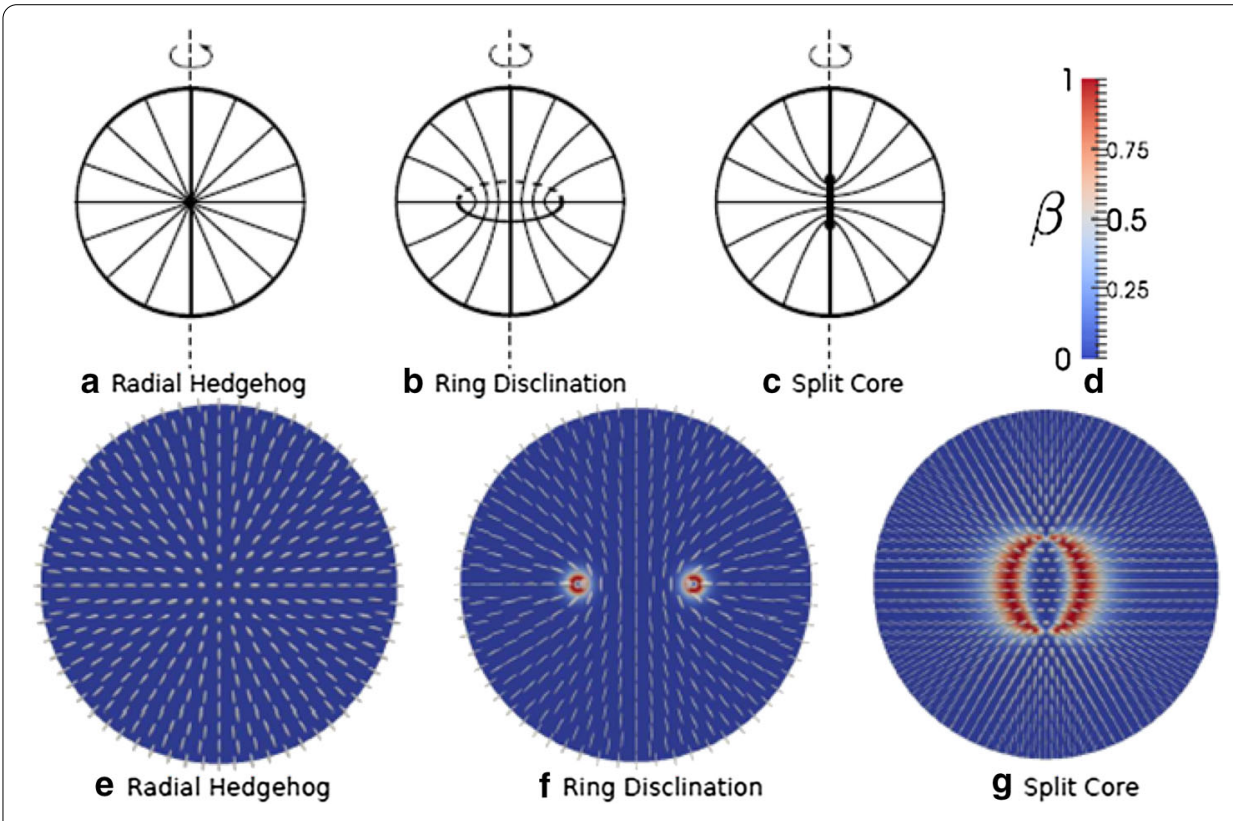

Fig. 1 Three possible configurations under $k=+1$. a-c show, respectively, qualitative alignment directions of hedgehog, ring and split-core solution on the $x-z$ plane. $\mathbf{e}-\mathbf{g}$ show corresponding distribution of $\beta$ on the $x-z$ plane. This figure is from [10]

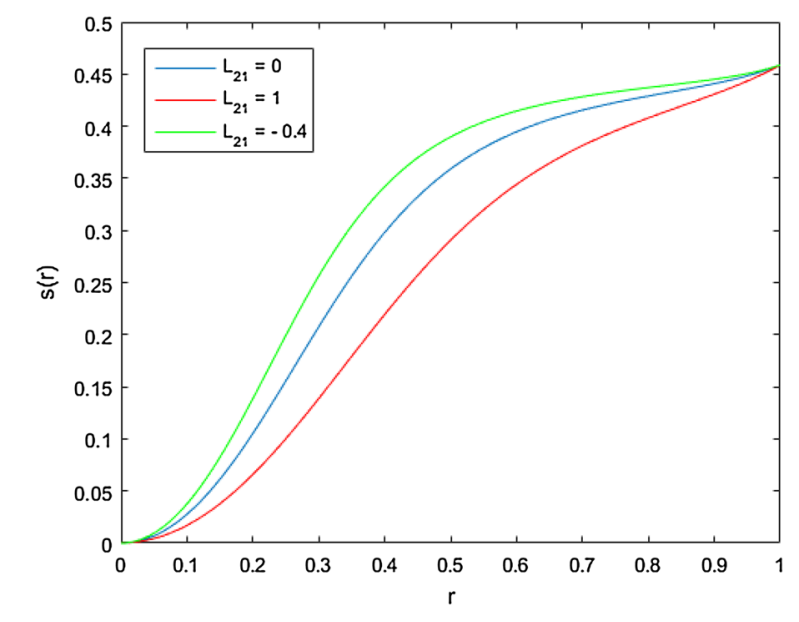

Fig. 2 Curves of radial scalar function $s(r)$ for different $L_{21}$. Other coefficients are $t=-0.5, \varepsilon=0.2$. The orders of Zernike polynomials are $N=64, L=64, M=32$, and we take penalty coefficient $\eta$ as to $10^{5}$, forcing the penalty energy $F_{p}$ to be $<10^{-15}$

- One can also obtain a ring solution and a split-core solution for $L_{21} \neq 0$ similar to the case of $L_{21}=0$. Our numeral results indicate that they are still rotationally symmetric. To verify it, we define the error function

$$
\operatorname{err}(r, \theta, \varphi)=\left|Q(r, \theta, \varphi)-P_{\varphi} Q(r, \theta, 0) P_{\varphi}^{\mathrm{T}}\right| .
$$

If $Q$ is axially symmetric, err will be 0 everywhere. Due to the existence of numerical error, a small maximum of error function will support the axial symmetry. Numerical verifications on the axial symmetry are listed in Table 1. 
Table 1 Numerical verifications of rotational symmetries of the ring and the split-core solutions with nonzero $L_{21}$ for homeotropic anchoring

\begin{tabular}{|c|c|c|c|c|c|c|c|}
\hline \multicolumn{4}{|c|}{$t=0.3, \varepsilon=0.1, L_{21}=3$} & \multicolumn{4}{|c|}{$t=-3, \varepsilon=0.2, L_{21}=-0.2$} \\
\hline $\mathrm{N}$ & $\mathrm{L}$ & $M$ & $\|\operatorname{err}\|_{L}^{\infty}$ & $\mathrm{N}$ & $\mathrm{L}$ & $\mathrm{M}$ & $\|e r r\|_{\mathbf{L}}^{\infty}$ \\
\hline \multicolumn{8}{|c|}{ Ring } \\
\hline 32 & 16 & 4 & $6.06 \mathrm{e}-7$ & 32 & 16 & 4 & $1.70 e-6$ \\
\hline 32 & 16 & 8 & $4.85 e-7$ & 32 & 16 & 8 & $3.70 e-6$ \\
\hline 32 & 32 & 16 & $4.72 \mathrm{e}-7$ & 32 & 32 & 16 & $2.22 \mathrm{e}-6$ \\
\hline 32 & 32 & 32 & $4.71 e-7$ & 32 & 32 & 32 & $2.22 \mathrm{e}-6$ \\
\hline \multicolumn{4}{|c|}{$t=-7, \varepsilon=0.2, L_{21}=0.3$} & \multicolumn{4}{|c|}{$t=-7, \varepsilon=0.2, L_{21}=-0.1$} \\
\hline $\mathrm{N}$ & $\mathrm{L}$ & $\mathrm{M}$ & $\|\operatorname{err}\|_{L}^{\infty}$ & $\mathrm{N}$ & $\mathrm{L}$ & M & $\|\operatorname{err}\|_{L}^{\infty}$ \\
\hline \multicolumn{8}{|c|}{ Split-core } \\
\hline 32 & 16 & 4 & $3.03 e-6$ & 32 & 16 & 4 & $8.37 e-7$ \\
\hline 32 & 16 & 8 & $1.64 \mathrm{e}-6$ & 32 & 16 & 8 & $1.30 \mathrm{e}-6$ \\
\hline 32 & 32 & 16 & $9.71 e-6$ & 32 & 32 & 16 & $1.07 e-6$ \\
\hline 32 & 32 & 32 & $2.13 e-6$ & 32 & 32 & 32 & $1.06 \mathrm{e}-6$ \\
\hline
\end{tabular}

We have also obtained phase diagrams for different $L_{21}$, which is shown in Fig. 3. This implies that $L_{21}$ affects the stability of radial hedgehog and ring solution continuously. It is worth remarking that the equilibrium solutions under homeotropic anchoring condition have also been studied in [20] under the assumption of axial symmetry. Our full 3D simulation verifies the validity of this assumption.

\subsection{Mirror-homeotropic anchoring condition}

4.2.1 The case of $L_{21}=0$

The case of $L_{21}=0$ in mirror-homeotropic anchoring condition actually shares the "same" behavior with homeotropic case, due to a simple argument: If $Q(\mathbf{x})$ is an equilibrium solution/a minimizer for homeotropic anchoring condition, then

$$
\hat{Q}(\mathbf{x})=M_{z} Q M_{z}
$$

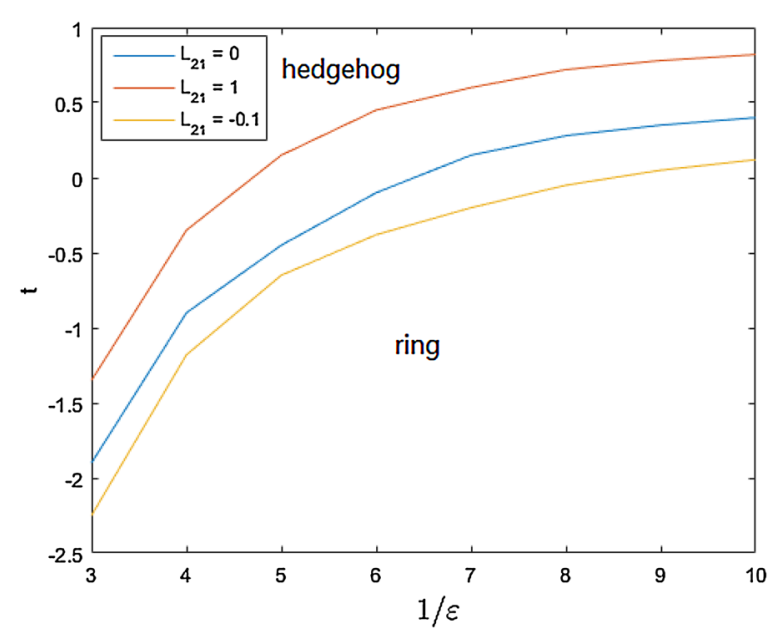

Fig. 3 Phase diagram of radial hedgehog and ring for homeotropic anchoring condition with different $L_{21}$ 
is an equilibrium solution/a minimizer for mirror-homeotropic anchoring condition, and vise versa. Here $M_{z}=\operatorname{diag}\{1,1,-1\}$.

Therefore, we can find two stable solutions analogue to the radial hedgehog and ring solution in homeotropic anchoring case and a meta-stable solution similar to split-core, respectively:

- Hyperbolic hedgehog solution: This solution is a "mirror" version of the radial hedgehog. Thus, it is uniaxial everywhere (except an isolated isotropic point in the center), and the order parameter is a radial symmetric function. Precisely, it has the following form

$$
Q(\mathbf{x})=s(r)\left(\mathbf{m} \otimes \mathbf{m}-\frac{1}{3} I\right), \quad \mathbf{m}=(x, y,-z) / r .
$$

However, the orientation directors in this solution are no longer aligned as "hedgehog," so we call it hyperbolic hedgehog. This solution is illustrated in Fig. 4a.

- The ring solution and the split-core solution of mirror-homeotropic anchoring case are quite similar to the corresponding solutions of homeotropic anchoring case, with the same distributions on $\beta$ but different eigenvectors. These solutions are illustrated in Fig. 4.

\subsubsection{The case of $L_{21}>0$}

When $L_{21}>0$, numerical simulation reveals two different solutions, which are splitcore and ring disclination (see Fig. 4b, c). These two kinds of solutions preserve the axial symmetry, which is verified by the numerical results in Table 2 .

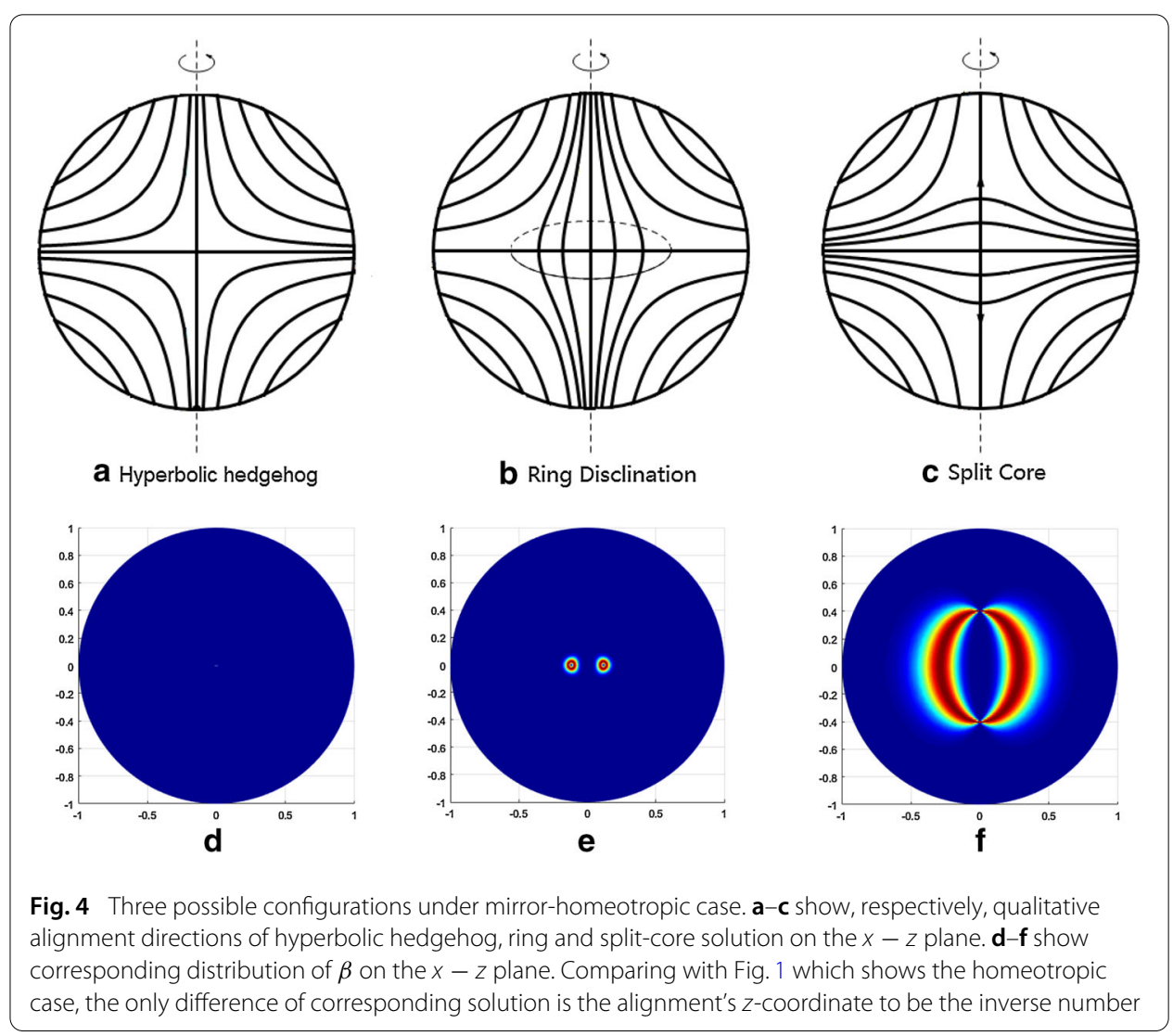


Table 2 Numerical verification of ring's and split-core's axial symmetry with mirror-homeotropic anchoring condition

\begin{tabular}{|c|c|c|c|c|c|c|c|}
\hline \multicolumn{4}{|c|}{$t=-2, \boldsymbol{\varepsilon}=.050, L_{21}=2$} & \multicolumn{4}{|c|}{$t=.50, \varepsilon=.10, L_{21}=2$} \\
\hline $\mathrm{N}$ & $\mathrm{L}$ & M & $\|\operatorname{err}\|_{L}^{\infty}$ & $\mathrm{N}$ & $\mathrm{L}$ & M & $\|\operatorname{err}\|_{L}^{\infty}$ \\
\hline \multicolumn{8}{|c|}{ Split-core } \\
\hline 32 & 16 & 4 & $1.79 e-6$ & 32 & 16 & 4 & $6.62 e-7$ \\
\hline 32 & 16 & 8 & $1.92 \mathrm{e}-6$ & 32 & 16 & 8 & $6.47 e-7$ \\
\hline 32 & 32 & 16 & $2.04 \mathrm{e}-6$ & 32 & 32 & 16 & $4.38 \mathrm{e}-7$ \\
\hline 32 & 32 & 32 & $2.10 e-6$ & 32 & 32 & 32 & $4.38 e-7$ \\
\hline \multicolumn{4}{|c|}{$t=-2, \varepsilon=.10, L_{21}=-.20$} & \multicolumn{4}{|c|}{$t=-8, \boldsymbol{\varepsilon}=.10, L_{21}=.50$} \\
\hline $\mathrm{N}$ & $\mathrm{L}$ & $M$ & $\|$ err $\| L^{\infty}$ & $\mathrm{N}$ & $\mathrm{L}$ & M & $\|\operatorname{err}\| L^{\infty}$ \\
\hline \multicolumn{8}{|c|}{ Ring } \\
\hline 32 & 16 & 4 & $1.67 e-6$ & 32 & 16 & 4 & $2.61 e-5$ \\
\hline 32 & 16 & 8 & $1.67 e-6$ & 32 & 16 & 8 & $2.61 e-5$ \\
\hline 32 & 32 & 16 & $1.47 e-6$ & 32 & 32 & 16 & $2.60 e-5$ \\
\hline 32 & 32 & 32 & $1.52 \mathrm{e}-6$ & 32 & 32 & 32 & $2.57 e-5$ \\
\hline
\end{tabular}

As for stability, phase diagrams are shown in Fig. 5 . Here for large $t$ and $\varepsilon$, split-core solution is stable, and ring solution is energetically favored if taking small $t$ and $\varepsilon$. We remark that when $L_{21}>0$ we cannot find the hyperbolic hedgehog solution. Actually, when $L_{21} \neq 0$, it can be proved that such uniaxial solution cannot be an equilibrium solution of the Landau-de Gennes energy functional. We will carry out a detailed analysis in the next section.

\subsubsection{The case of $L_{21}<0$}

For $L_{21}<0$ case, our numerical results suggest that there exists only one stable equilibrium solution, ring solution, whatever coefficients $t$ and $\varepsilon$ vary. Not only hyperbolic hedgehog solution but also split-core solution disappears.

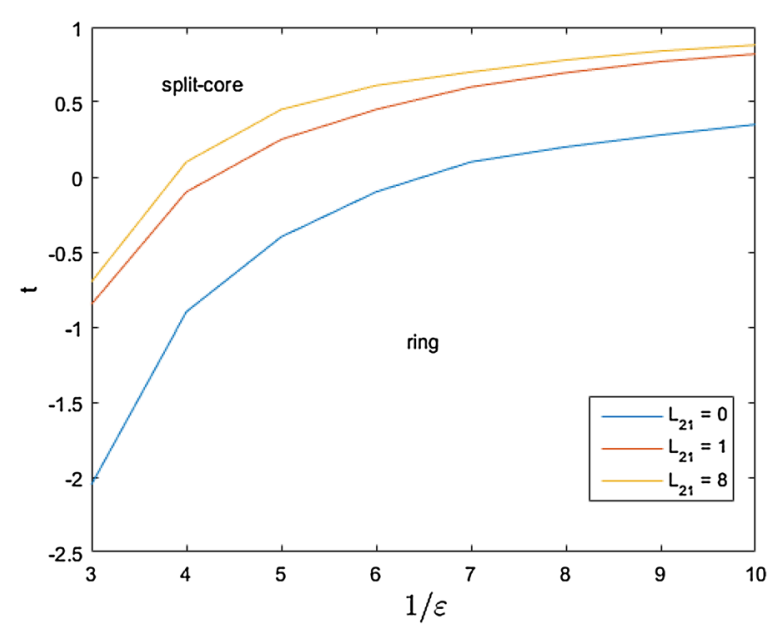

Fig. 5 Phase diagram of split-core/hyperbolic hedgehog and ring solution for different $L_{21} \geq 0$ with mirror-homeotropic anchoring condition. When $L_{21}=0$, the notation "split-core" indicates hyperbolic hedgehog. The partition of different solutions is based on the lowest energy of all the possible configurations. Here, there is no phase diagram of $L_{21}<0$ because there is only ring solution 
Table 3 Numerical value of $Q(0)$ with parameters $t=0.2, \varepsilon=0.2$ and $t=-2, \varepsilon=0.05$

\begin{tabular}{|c|c|c|c|c|c|c|}
\hline \multicolumn{7}{|c|}{$\mathrm{t}=0.2, \varepsilon=0.2(\mathrm{~N}=64, \mathrm{~L}=32, \mathrm{M}=32)$} \\
\hline $\mathbf{L}_{21}$ & $\mathrm{Q}_{11}$ & $\mathrm{Q}_{12}$ & $\mathrm{Q}_{13}$ & $\mathrm{Q}_{22}$ & $\mathrm{Q}_{23}$ & $\|\nabla \mathbf{F}\|$ \\
\hline-0.10 & $-3.88 e-2$ & $5.33 e-12$ & $-6.9 e-14$ & $-3.88 e-2$ & $-3 e-15$ & $9.85 e-5$ \\
\hline-0.05 & $-1.68 e-2$ & $3.19 e-13$ & $2 e-15$ & $-1.68 e-2$ & $-2 e-15$ & $9.34 \mathrm{e}-5$ \\
\hline-0.01 & $-3.04 e-3$ & $1.11 \mathrm{e}-13$ & $-6 e-15$ & $-3.04 e-3$ & $1 e-15$ & $1.00 e-5$ \\
\hline 0 & $-8.57 e-6$ & $-3.27 e-12$ & $-6.0 e-14$ & $-8.57 e-6$ & $-3.1 e-14$ & $1.26 \mathrm{e}-4$ \\
\hline 0.01 & $2.93 e-3$ & $1.57 e-13$ & $-1.1 e-14$ & $2.93 e-3$ & 0 & $3.71 e-4$ \\
\hline 0.05 & $1.37 e-2$ & $2.78 \mathrm{e}-13$ & $1.76 e-13$ & $1.37 e-2$ & $-5.51 e-13$ & $1.04 \mathrm{e}-4$ \\
\hline 0.10 & $2.52 \mathrm{e}-2$ & $2.31 \mathrm{e}-12$ & $2.1 e-14$ & $2.52 \mathrm{e}-2$ & $-5 e-15$ & $1.96 \mathrm{e}-4$ \\
\hline \multicolumn{7}{|c|}{$\mathrm{t}=-2, \varepsilon=0.05(\mathrm{~N}=64, \mathrm{~L}=32, \mathrm{M}=32)$} \\
\hline $\mathrm{L}_{21}$ & $\mathrm{Q}_{11}$ & $\mathrm{Q}_{12}$ & $\mathrm{Q}_{13}$ & $\mathrm{Q}_{22}$ & $\mathrm{Q}_{23}$ & $\|\nabla \mathbf{F}\|$ \\
\hline-0.10 & $-4.75 e-2$ & $-7.06 e-11$ & $2.07 e-13$ & $-4.75 e-2$ & $-1.42 e-12$ & $1.53 e-4$ \\
\hline-0.05 & $-2.10 e-2$ & $-1.31 \mathrm{e}-11$ & $-2.86 e-13$ & $-2.10 e-2$ & $-1.16 e-13$ & $2.06 e-4$ \\
\hline-0.01 & $-3.93 e-3$ & $-2.59 e-12$ & $1.04 \mathrm{e}-13$ & $-3.93 e-3$ & $7.3 e-14$ & $1.92 \mathrm{e}-4$ \\
\hline 0 & $-6.99 e-6$ & $-1.26 e-11$ & $4.93 e-13$ & $-6.99 e-6$ & $-5.53 e-13$ & $2.93 e-4$ \\
\hline 0.01 & $3.72 \mathrm{e}-3$ & $-4.16 e-12$ & $2.06 e-13$ & $3.72 \mathrm{e}-3$ & $9.0 e-14$ & $1.37 e-4$ \\
\hline 0.05 & $1.81 \mathrm{e}-2$ & $6.62 e-13$ & $4.56 e-13$ & $1.81 \mathrm{e}-2$ & $2.65 e-13$ & $3.85 e-4$ \\
\hline 0.10 & $3.41 \mathrm{e}-2$ & $5.23 e-13$ & $6.8 e-14$ & $3.41 \mathrm{e}-2$ & $7.5 e-14$ & $5.63 e-4$ \\
\hline
\end{tabular}

The rows of $L_{21}=0$ represent stable hyperbolic hedgehog solutions which serve as initial values to obtain split-core $\left(L_{2}>0\right)$ or ring $\left(L_{2}<0\right)$ solutions

This interesting phenomenon leads to a natural conjecture that there exists at least one split-core solution, which is stable when $L_{21}>0$; however, all split-core solutions are unstable when $L_{21}<0$ and are at most meta-stable for $L_{21}=0$.

\subsubsection{More detailed transition behavior of hyperbolic hedgehog solution near $L_{21}=0$}

According to our aforementioned numerical results, we find out that there is no hyperbolic hedgehog solution when $L_{21} \neq 0$. Now, we study how the hyperbolic hedgehog solution evolves when $L_{21}$ varies near zero. For this, we focus on the values of all components of $Q$-tensor at the center of ball, namely $Q(0)$. From the definitions of three basic solutions, we know that: $Q(0)=0$ for hyperbolic hedgehog solution, $Q(0)$ is positive uniaxial for ring solution and $Q(0)$ is negative uniaxial for split-core solution.

The values of $Q(0)$ for minimizers with respect to different $L_{21}$ are presented in Table 3 for two different choices of $(t, \varepsilon)$. All these results are obtained by an iterative method with initial value taken to be the hyperbolic hedgehog solution. It can be observed that $Q(0)$ is always uniaxial when $L_{21} \neq 0$. In addition, $Q(0)$ is negative uniaxial if $L_{21}>0$ and positive uniaxial if $L_{21}<0$. These results reveal that a positive $L_{21}$ may break the hyperbolic hedgehog into a split-core and a negative $L_{21}$ can broaden it into a ring disclination.

We also investigate the qualitative relationship between the size of biaxial region and $L_{21}$. We call the distance between two isotropic points "split length." The relationship between split length and $L_{21}$ is shown in Figs. 6 and 7. It can be observed that split length is proportional to $L_{21}$ near $L_{21}=0$. Thus, the hyperbolic hedgehog solution for $L_{21}$ can be viewed as a special split-core solution with zero split length. 

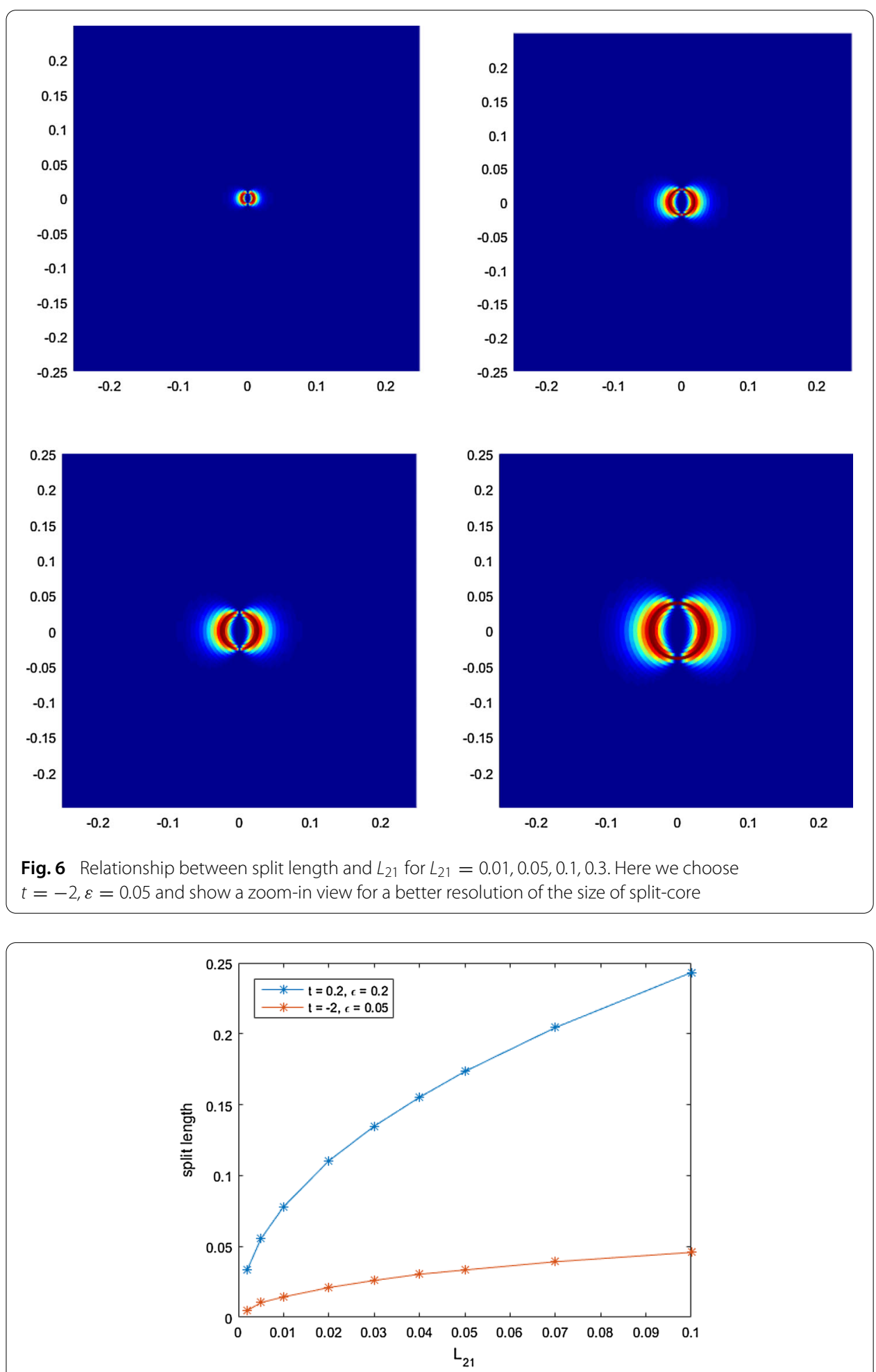

Fig. 7 Relationship between split length and $L_{21}$ 


\section{Nonexistence of uniaxial hyperbolic hedgehog solutions for} mirror-homeotropic anchoring condition with $L_{\mathbf{2 1}} \neq 0$

In this section, we take $\varepsilon=1$ and work on $\Omega=B_{R}(0)$ for convenience. Then, the EulerLagrange equation to the Landau-de Gennes energy reads as

$$
\begin{aligned}
& \Delta Q_{i j}+\frac{L_{21}}{2}\left(Q_{i k, k j}+Q_{j k, k i}-\frac{2}{3} \delta_{i j} Q_{k l, k l}\right) \\
& \quad=-t Q_{i j}-3 \sqrt{6}\left(Q_{i k} Q_{k j}-\frac{\delta_{i j}}{3} \operatorname{tr} Q^{2}\right)+2 Q_{i j} \operatorname{tr} Q^{2} .
\end{aligned}
$$

For the homeotropic boundary condition, there is a radial hedgehog solution to (26) in $B_{R}(0)$ explicitly given by

$$
Q(\mathbf{x})=\sqrt{\frac{3}{2}} h(r)\left(\frac{\mathbf{x}}{r} \otimes \frac{\mathbf{x}}{r}-\frac{1}{3} I\right)
$$

where $h(r)$ satisfies the following equation:

$$
\left(1+\frac{2}{3} L_{21}\right)\left(h^{\prime \prime}+\frac{2}{r} h^{\prime}-\frac{6}{r^{2}} h\right)=-t h-3 h^{2}+2 h^{3}, \quad h(0)=0, h(R)=\sqrt{\frac{2}{3}} s_{+} .
$$

In this section, we will show that the hyperbolic hedgehog solution cannot be a solution to (26) with mirror-homeotropic boundary condition.

Assume that

$$
Q(\mathbf{x})=\sqrt{\frac{3}{2}} h(r)\left(\mathbf{m}(\mathbf{x}) \otimes \mathbf{m}(\mathbf{x})-\frac{1}{3} I\right), \quad \mathbf{m}(\mathbf{x})=\frac{1}{r}(x, y,-z),
$$

is a solution to the Euler-Lagrange equation (26). Substituting (29)-(26), we have

$$
\frac{1}{\sqrt{6}} L_{21} P=\left(-h^{\prime \prime}-\frac{2}{r} h^{\prime}+\frac{6}{r^{2}} h-t h-3 h^{2}+2 h^{3}\right)\left(\mathbf{m}(\mathbf{x}) \otimes \mathbf{m}(\mathbf{x})-\frac{1}{3} I\right),
$$

in which

$$
P_{i j}=Q_{i k, k j}+Q_{j k, k i}-\frac{2}{3} \delta_{i j} Q_{k l, k l} .
$$

Notice that

$$
\nabla\left(\frac{h}{r^{2}}\right)=\left(\frac{h^{\prime}}{r^{3}}-\frac{2 h}{r^{4}}\right)(x, y, z)
$$

we have

$$
\begin{aligned}
\frac{\partial^{2}}{\partial x^{2}}\left(\frac{h}{r^{2}}\right) & =\frac{x^{2}}{r^{4}} h^{\prime \prime}+\frac{r^{2}-5 x^{2}}{r^{5}} h^{\prime}-\frac{2 r^{2}-8 x^{2}}{r^{6}} h, \\
\frac{\partial^{2}}{\partial y^{2}}\left(\frac{h}{r^{2}}\right) & =\frac{y^{2}}{r^{4}} h^{\prime \prime}+\frac{r^{2}-5 y^{2}}{r^{5}} h^{\prime}-\frac{2 r^{2}-8 y^{2}}{r^{6}} h, \\
\frac{\partial^{2}}{\partial z^{2}}\left(\frac{h}{r^{2}}\right) & =\frac{z^{2}}{r^{4}} h^{\prime \prime}+\frac{r^{2}-5 z^{2}}{r^{5}} h^{\prime}-\frac{2 r^{2}-8 z^{2}}{r^{6}} h, \\
\frac{\partial^{2}}{\partial x \partial y}\left(\frac{h}{r^{2}}\right) & =\frac{x y}{r^{4}} h^{\prime \prime}-\frac{5 x y}{r^{5}} h^{\prime}+\frac{8 x y}{r^{6}} h,
\end{aligned}
$$




$$
\begin{aligned}
& \frac{\partial^{2}}{\partial x \partial z}\left(\frac{h}{r^{2}}\right)=\frac{x z}{r^{4}} h^{\prime \prime}-\frac{5 x z}{r^{5}} h^{\prime}+\frac{8 x z}{r^{6}} h, \\
& \frac{\partial^{2}}{\partial y \partial z}\left(\frac{h}{r^{2}}\right)=\frac{y z}{r^{4}} h^{\prime \prime}-\frac{5 y z}{r^{5}} h^{\prime}+\frac{8 y z}{r^{6}} h .
\end{aligned}
$$

Therefore, applying the chain rule

$$
(u v)_{i j}=u_{i j} v+u_{i} v_{j}+u_{j} v_{i}+u v_{i j}
$$

we obtain

$$
\begin{aligned}
& \sqrt{\frac{2}{3}} Q_{11,12}=\left(\frac{x^{3} y}{r^{4}}-\frac{x y}{3 r^{2}}\right) h^{\prime \prime}+\left(-\frac{5 x^{3} y}{r^{5}}+\frac{7 x y}{3 r^{3}}\right) h^{\prime}+\left(\frac{8 x^{3} y}{r^{6}}-\frac{4 x y}{r^{4}}\right) h, \\
& \sqrt{\frac{2}{3}} Q_{12,22}=\left(\frac{x y^{3}}{r^{4}}\right) h^{\prime \prime}+\left(\frac{3 x y}{r^{3}}-\frac{5 x y^{3}}{r^{5}}\right) h^{\prime}+\left(-\frac{6 x y}{r^{4}}+\frac{8 x y^{3}}{r^{6}}\right) h, \\
& \sqrt{\frac{2}{3}} Q_{13,32}=\left(-\frac{x y z^{2}}{r^{4}}\right) h^{\prime \prime}+\left(\frac{5 x y z^{2}}{r^{5}}-\frac{x y}{r^{3}}\right) h^{\prime}+\left(-\frac{8 x y z^{2}}{r^{6}}+\frac{2 x y}{r^{4}}\right) h, \\
& \sqrt{\frac{2}{3}} Q_{21,11}=\left(\frac{x^{3} y}{r^{4}}\right) h^{\prime \prime}+\left(\frac{3 x y}{r^{3}}-\frac{5 x^{3} y}{r^{5}}\right) h^{\prime}+\left(-\frac{6 x y}{r^{4}}+\frac{8 x^{3} y}{r^{6}}\right) h, \\
& \sqrt{\frac{2}{3}} Q_{22,21}=\left(\frac{y^{3} x}{r^{4}}-\frac{y x}{3 r^{2}}\right) h^{\prime \prime}+\left(-\frac{5 y^{3} x}{r^{5}}+\frac{7 y x}{3 r^{3}}\right) h^{\prime}+\left(\frac{8 y^{3} x}{r^{6}}-\frac{4 y x}{r^{4}}\right) h, \\
& \sqrt{\frac{2}{3}} Q_{23,31}=\left(-\frac{x y z^{2}}{r^{4}}\right) h^{\prime \prime}+\left(\frac{5 x y z^{2}}{r^{5}}-\frac{x y}{r^{3}}\right) h^{\prime}+\left(-\frac{8 x y z^{2}}{r^{6}}+\frac{2 x y}{r^{4}}\right) h,
\end{aligned}
$$

and

$$
\begin{aligned}
& \sqrt{\frac{2}{3}} Q_{11,13}=\left(\frac{x^{3} z}{r^{4}}-\frac{x z}{3 r^{2}}\right) h^{\prime \prime}+\left(-\frac{5 x^{3} z}{r^{5}}+\frac{7 x z}{3 r^{3}}\right) h^{\prime}+\left(\frac{8 x^{3} z}{r^{6}}-\frac{4 x z}{r^{4}}\right) h, \\
& \sqrt{\frac{2}{3}} Q_{12,23}=\left(\frac{x y^{2} z}{r^{4}}\right) h^{\prime \prime}+\left(-\frac{5 x y^{2} z}{r^{5}}+\frac{x z}{r^{3}}\right) h^{\prime}+\left(\frac{8 x y^{2} z}{r^{6}}-\frac{2 x z}{r^{4}}\right) h, \\
& \sqrt{\frac{2}{3}} Q_{13,33}=\left(-\frac{x z^{3}}{r^{4}}\right) h^{\prime \prime}+\left(-\frac{3 x z}{r^{3}}+\frac{5 x z^{3}}{r^{5}}\right) h^{\prime}+\left(\frac{6 x z}{r^{4}}-\frac{8 x z^{3}}{r^{6}}\right) h, \\
& \sqrt{\frac{2}{3}} Q_{31,11}=\left(-\frac{x^{3} z}{r^{4}}\right) h^{\prime \prime}+\left(-\frac{3 x z}{r^{3}}+\frac{5 x^{3} z}{r^{5}}\right) h^{\prime}+\left(\frac{6 x z}{r^{4}}-\frac{8 x^{3} z}{r^{6}}\right) h, \\
& \sqrt{\frac{2}{3}} Q_{32,21}=\left(-\frac{x y^{2} z}{r^{4}}\right) h^{\prime \prime}+\left(\frac{5 x y^{2} z}{r^{5}}-\frac{x z}{r^{3}}\right) h^{\prime}+\left(-\frac{8 x y^{2} z}{r^{6}}+\frac{2 x z}{r^{4}}\right) h, \\
& \sqrt{\frac{2}{3}} Q_{33,31}=\left(\frac{z^{3} x}{r^{4}}-\frac{z x}{3 r^{2}}\right) h^{\prime \prime}+\left(-\frac{5 z^{3} x}{r^{5}}+\frac{7 z x}{3 r^{3}}\right) h^{\prime}+\left(\frac{8 z^{3} x}{r^{6}}-\frac{4 z x}{r^{4}}\right) h .
\end{aligned}
$$

Hence, we arrive at

$$
\begin{aligned}
& \sqrt{\frac{2}{3}} P_{12}=\left(\frac{4 x y}{3 r^{2}}-\frac{4 x y z^{2}}{r^{4}}\right) h^{\prime \prime}+\left(-\frac{4 x y}{3 r^{3}}+\frac{20 x y z^{2}}{r^{5}}\right) h^{\prime}+\left(-\frac{32 x y z^{2}}{r^{6}}\right) h, \\
& \sqrt{\frac{2}{3}} P_{13}=\left(-\frac{2 x z}{3 r^{2}}\right) h^{\prime \prime}+\left(-\frac{4 x z}{3 r^{3}}\right) h^{\prime}+\left(\frac{4 x z}{r^{4}}\right) h .
\end{aligned}
$$


In order to show that the Euler-Lagrange equation (26) cannot hold, we consider the equations for $i=1, j=2$ and $i=1, j=3$,

$$
\begin{gathered}
\left(h^{\prime \prime}+\frac{2}{r} h^{\prime}-\frac{6}{r^{2}} h\right) \frac{x y}{r^{2}}+\frac{1}{\sqrt{6}} L_{21} P_{12}=\left(-t h-3 h^{2}+2 h^{3}\right) \frac{x y}{r^{2}} \\
\left(h^{\prime \prime}+\frac{2}{r} h^{\prime}-\frac{6}{r^{2}} h\right) \frac{-x z}{r^{2}}+\frac{1}{\sqrt{6}} L_{21} P_{13}=\left(-t h-3 h^{2}+2 h^{3}\right) \frac{-x z}{r^{2}} .
\end{gathered}
$$

Compare this two ODEs, we obtain

$$
P_{12} \frac{r^{2}}{x y}=-P_{13} \frac{r^{2}}{x z}
$$

which is equivalent to

$$
\left(\frac{4}{3}-\frac{4 z^{2}}{r^{2}}\right) h^{\prime \prime}+\left(-\frac{4}{3}+\frac{20 z^{2}}{r^{2}}\right) \frac{1}{r} h^{\prime}+\left(-\frac{32 z^{2}}{r^{2}}\right) \frac{h}{r^{2}}=\frac{2}{3} h^{\prime \prime}+\frac{4}{3} \frac{1}{r} h^{\prime}-4 \frac{h}{r^{2}} .
$$

Since the above equation holds for all $z$ and $r$, we have

$$
\begin{aligned}
& h^{\prime \prime}-\frac{4}{r} h^{\prime}+\frac{6 h}{r^{2}}=0, \\
& h^{\prime \prime}-\frac{5}{r} h^{\prime}+\frac{8 h}{r^{2}}=0 .
\end{aligned}
$$

Combined with the boundary conditions, these two ODEs can be explicitly solved as

$$
h(r)=\sqrt{\frac{2}{3}} \frac{s_{+}}{R^{2}} r^{2}
$$

However, one can easily check that (35) is not the solution of (33). Such contradiction indicates that the hyperbolic hedgehog cannot be a solution to (26).

\section{Discussion and conclusion}

In this paper, we investigate the effect of anisotropic energy to the local structure of point defects in a three-dimensional ball. Both homeotropic and mirror-homeotropic anchoring conditions, which correspond to degree +1 and -1 point defects, respectively, are considered.

Our numerical results reveal that the anisotropic energy will affect the phase behavior significantly for the mirror-homeotropic anchoring condition even if the absolute value of anisotropic elastic coefficient $\left|L_{21}\right|$ is small. When $L_{21} \neq 0$, there is no uniaxial solution with radial symmetric order parameter which may be stable minimizer for certain parameters. More precisely, the uniaxial solution will deform into a split-core solution for $L_{21}>0$ and into a ring solution for $L_{21}<0$ in high-temperature region. This is very different from the homeotropic anchoring case, in which the three basic configurations still exist and their stabilities are not essentially affected by $L_{21}$. In particular, the radially symmetric solution is preserved when $L_{21} \neq 0$ in homeotropic anchoring case.

We further perform analysis on the hyperbolic hedgehog solution for the mirrorhomeotropic anchoring. We prove that the hyperbolic hedgehog solution cannot be a solution to the Euler-Lagrange equation when $L_{21} \neq 0$. Based on this result, it is quite reasonable to make the following conjecture. 
Conjecture 1 Uniaxial solution with degree -1 cannot be a stable minimizer once anisotropic elastic energy is considered.

We have also found out that there exist stable split-core solutions for high temperature when $L_{21}>0$. This is different from the isotropic energy case, in which split-core solutions are shown to be only meta-stable. More interestingly, the stable split-core solution will reduce to hyperbolic hedgehog when $L_{21}$ goes to zero and will deform to the ring solution if $L_{21}$ decreases to be negative. This inspires us to make the following conjecture.

Conjecture 2 For the mirror-homeotropic anchoring condition, there exists stable splitcore solution for high temperature when $L_{21}$ is positive, but split-core solution is unstable everywhere once $L_{21}$ is negative.

As a final point, we remark that all the solutions obtained in this paper are axisymmetric although such a symmetric constrain is not imposed in our simulation. Whether there is any other solution without axisymmetry is worth being investigated. We leave it to future works.

\section{Author details}

${ }^{1}$ LMAM and School of Mathematical Science, Peking University, Beijing 100871, China, ${ }^{2}$ School of Mathematical Sciences, Zhejiang University, Hangzhou 310027, China.

\section{Acknowledgements}

The authors would like to thank the anonymous referees for their valuable comments. WW was supported by NSF of China under Grant 11501502 and "the Fundamental Research Funds for the Central Universities" 2016QNA3004. PZ was supported by NSF of China under Grants 11421101 and 11421110001.

\section{Appendix: Numerical results on general boundary conditions with $|\boldsymbol{k}| \geq \mathbf{2}$}

We also present some numerical results with general boundary conditions (10) with $|k| \geq$ 2. First, we emphasize that for such boundary conditions there is no difference with or without anisotropic elastic energy. However, there do exist some interesting phenomena related to such boundary conditions, so we state this part of results in "Appendix."

We only find ring-type solutions as stable equilibrium configurations for a broad range of parameters $\left(t, \varepsilon, L_{21}\right)$. The typical configurations of ring-type solutions for $k= \pm 2, \pm 3, \pm 4$ with $L_{21}=0$ are shown in Fig. 8 .

It can be observed that there are $|k|$ rings of defects with charge $\operatorname{sgn}(k) / 2$. In addition, these solutions admit axial symmetry with respect to $z$-axis and mirror symmetry with respect to $x-y$ plane. The anisotropic energy will not affect the result that ring-type solutions are the only observed stable ones.

Of course, we cannot exclude the existences of other type solutions, but it is reasonable to conjecture that other type solutions are at most meta-stable if they exist. For example, we can construct a uniaxial solution, which takes the following form:

$$
Q(\mathbf{x})=\sqrt{\frac{3}{2}} h(|\mathbf{x}|)\left(\mathbf{m}(\mathbf{x}) \otimes \mathbf{m}(\mathbf{x})-\frac{1}{3} I\right),
$$

with $h$ is given by the Euler-Lagrange equation of minimizing the energy function on $Q$ which has the form (36). Such solutions include only a point defect with degree $k$ at the center of the ball. It seems that this uniaxial solution could be stable for $k= \pm 1$ for large $t$ and $\varepsilon$ under isotropic case, but will never be stable for $|k| \geq 2$ if $R$ is large enough no matter how $t$ and $L_{21}$ vary. It may be an interesting issue to prove it analytically. 


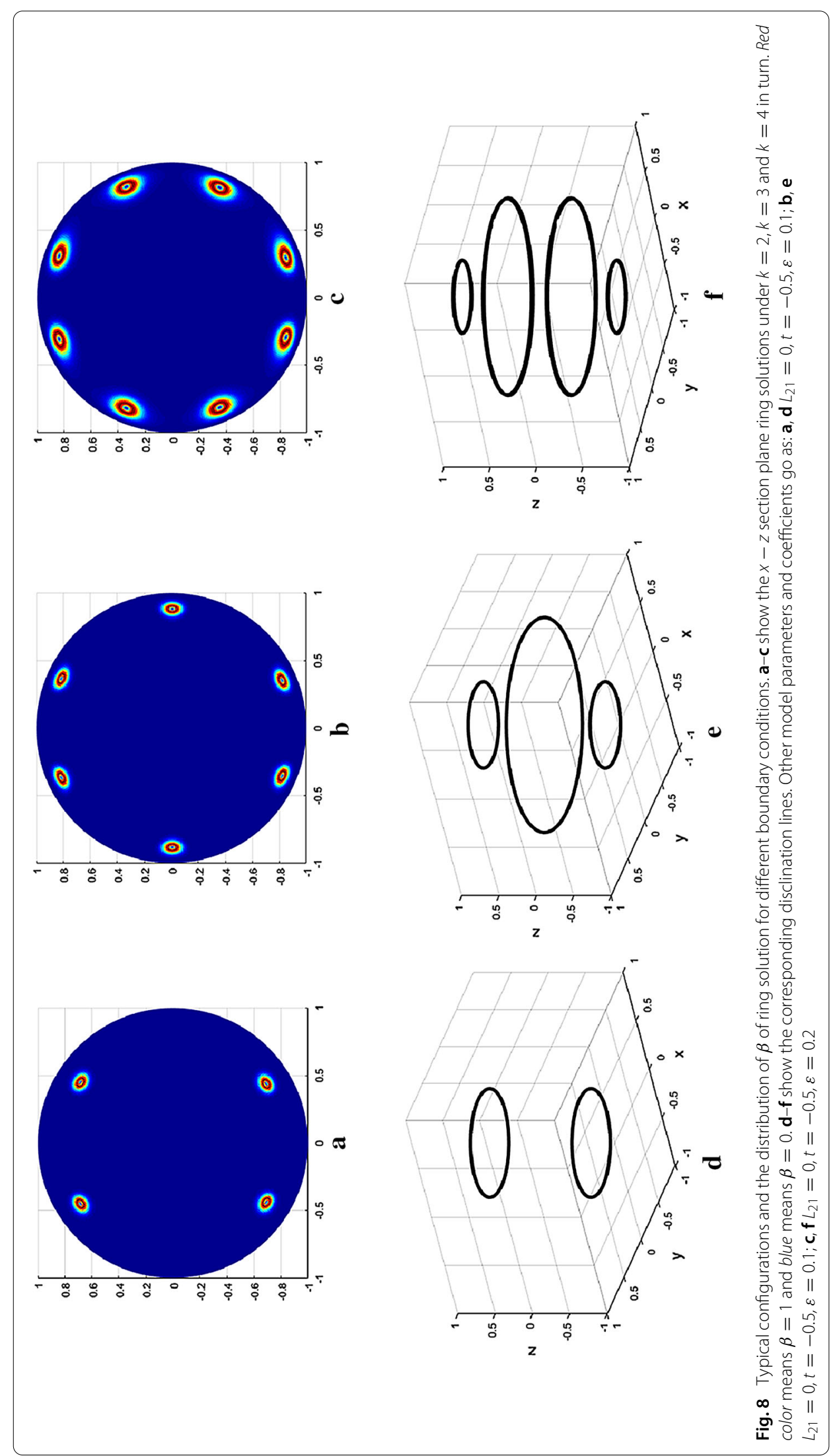


Received: 30 June 2016 Accepted: 30 December 2016

Published online: 10 March 2017

\section{References}

1. Avriel, M.: Nonlinear Programming: Analysis and Methods. Courier Dover Publications, Mineola (2003)

2. Ball, J.M., Zarnescu, A.: Orientability and energy minimization in liquid crystal models. Arch. Ration. Mech. Anal. 202, 493-535 (2011)

3. Canevari, G., Ramaswamy, M., Majumdar, A.: Radial symmetry on three-dimensional shells in the Landau-de Gennes theory. Phys. D 314, 18-34 (2016)

4. De Gennes, P.G.: The Physics of Liquid Crystals. Charendon, Oxford (1974)

5. Ericksen, J.: Liquid crystals with variable degree of orientation. Arch. Ration. Mech. Anal. 113, 97-120 (1991)

6. Fei, M.W., Wang, W., Zhang, P.W., Zhang, Z.F.: Dynamics of the nematic-isotropic sharp interface for the liquid crystal. SIAM J. Appl. Math. 75(4), 1700-1724 (2015)

7. Fratta, G.D., Robbins, J.M., Slastikov, V., Zarnescu, A.: Half-integer point defects in the Q-tensor theory of nematic liquid crystals. arXiv:1403.2566

8. Gartland, E.C., Mkaddem, S.: Instability of radial hedgehog configurations in nematic liquid crystals under Landau-de Gennes free-energy models. Phys. Rev. E 59, 563-567 (1999)

9. Hardt, R., Kinderlehrer, D., Lin, F.H.: Existence and partial regularity of static liquid crystal configurations. Commun. Math. Phys. 105(4), 547-570 (1986)

10. Hu, Y.C., Qu, Y., Zhang, P.W.: On the disclination lines of nematic liquid crystals. Commun. Comput. Phys. 19, 354-379 (2016)

11. Ignat, R., Nguyen, L., Slastikov, V., Zarnescu, A.: Stability of the melting hedgehog in the Landau-de Gennes theory of nematic liquid crystals. Arch. Ration. Mech. Anal. 215, 633-673 (2015)

12. Kinderlehrer, D., Ou, B., Walkington, N.J.: The elementary defects of the Oseen-Frank energy for a liquid crystal. C. R. Acad. Sci. Paris Serie I 316, 465-470 (1993)

13. Kralj, S., Virga, E.: Universal fine structure of nematic hedgehogs. J. Phys. A Math. Gen. 24, 829-838 (2001)

14. Lamy, X.: Some properties of the nematic radial hedgehog in the Landau-de Gennes theory. J. Math. Anal. Appl. 397, 586-594 (2013)

15. Lin, F.H., Liu, C.: Static and dynamic theories of liquid crystals. J. Partial Differ. Equ. 14, 289-330 (2001)

16. Majumdar, A., Zarnescu, A.: Landau-de Gennes theory of nematic liquid crystals: the Oseen-Frank limit and beyond. Arch. Ration. Mech. Anal. 196, 227-280 (2010)

17. Majumdar, A.: Equilibrium order parameters of liquid crystals in the Landau-de Gennes theory. Eur. J. Appl. Math. 21, $181-203(2010)$

18. Majumdar, A.: The radial-hedgehog solution in Landau-de Gennes' theory for nematic liquid crystals. Eur. J. Appl. Math. 23, 61-97 (2012)

19. Majumdar, A., Pisante, A., Henao, D.: Uniaxial versus biaxial character of nematic equilibria in three dimensions. arXiv:1312.3358

20. Mkaddem, S., Gartland, E.C.: Fine structure of defects in radial nematic droplets. Phys. Rev. E 62(5), 6694-6705 (2000)

21. Rosso, R., Virga, E.G.: Metastable nematic hedgehogs. J. Phys. A 29, 4247-4264 (1996)

22. Zernike, F.: Diffraction theory of the cut procedure and its improved form, the phase contrast method. Physica 1, 689-704 (1934)

\section{Submit your manuscript to a SpringerOpen ${ }^{\circ}$} journal and benefit from:

$\rightarrow$ Convenient online submission

- Rigorous peer review

- Immediate publication on acceptance

- Open access: articles freely available online

- High visibility within the field

- Retaining the copyright to your article

Submit your next manuscript at $\mathbf{s p r i n g e r o p e n . c o m ~}$ 